\title{
BMJ Open Temporal trends in out-of-hospital cardiac arrest survival outcomes between two metropolitan communities: Seoul-Osaka resuscitation study
}

\author{
Young Sun Ro, ${ }^{1}$ Sang Do Shin, ${ }^{2}$ Tetsuhisa Kitamura, ${ }^{3}$ Eui Jung Lee, ${ }^{2}$ \\ Kentaro Kajino, ${ }^{4}$ Kyoung Jun Song, ${ }^{2}$ Chika Nishiyama, ${ }^{5}$ So Yeon Kong, ${ }^{1}$ \\ Tomohiko Sakai, ${ }^{4}$ Tatsuya Nishiuchi, ${ }^{6}$ Yasuyuki Hayashi, ${ }^{7}$ Taku Iwami, ${ }^{8}$ \\ for Seoul-Osaka Resuscitation Study (SORS) Group
}

To cite: Ro YS, Shin SD, Kitamura T, et al. Temporal trends in out-of-hospital cardiac arrest survival outcomes between two metropolitan communities: Seoul-Osaka resuscitation study. BMJ Open 2015;5: e007626. doi:10.1136/ bmjopen-2015-007626

- Prepublication history for this paper is available online. To view these files please visit the journal online (http://dx.doi.org/10.1136/ bmjopen-2015-007626).

Received 11 January 2015 Revised 2 April 2015 Accepted 28 April 2015

CrossMark

For numbered affiliations see end of article.

Correspondence to

Taku Iwami;

iwamit@e-mail.jp

\section{ABSTRACT}

Objectives: The objective of this study was to compare the temporal trends in survival after out-ofhospital cardiac arrest (OHCA) between two large metropolitan communities in Asia and evaluate the factors affecting survival after OHCA.

Design: A population-based prospective observational study.

Setting: The Cardiovascular Disease Surveillance (CAVAS) project in Seoul and the Utstein Osaka Project in Osaka.

Participants: A total of 36292 resuscitationattempted OHCAs with cardiac aetiology from 2006 to 2011 in Seoul and Osaka (11 082 in Seoul and 25210 in Osaka).

Primary outcome measures: The primary outcome was neurologically favourable survival. Trend analysis and multivariable Poisson regression models were conducted to evaluate the temporal trends in survival of two communities.

Results: During the study period, the overall neurologically favourable survival was $2.6 \%$ in Seoul and $4.6 \%$ in Osaka $(\mathrm{p}<0.01)$. In both communities, bystander cardiopulmonary resuscitation (CPR) rates increased significantly from 2006 to 2011 (from $0.1 \%$ to $13.1 \%$ in Seoul and from $33.3 \%$ to $41.7 \%$ in Osaka). OHCAs that occurred in public places increased in Seoul $(12.5 \%$ to $20.1 \%, p$ for trend $<0.01)$ and decreased in Osaka ( $13.5 \%$ to $10.5 \%$, p for trend $<0.01)$. The proportion of OHCAs defibrillated by emergency medical service (EMS) providers was only $0.4 \%$ in 2006 but increased to $17.5 \%$ in 2011 in Seoul, whereas the proportion in Osaka decreased from $17.7 \%$ to $13.7 \%$ (both $p$ for trend $<0.01$ ). Ageadjusted and gender-adjusted rates of neurologically favourable survival increased significantly in Seoul from $1.4 \%$ in 2006 to $4.3 \%$ in 2011 (adjusted rate ratio per year, 1.17; $p$ for trend $<0.01$ ), whereas no significant improvement was observed in Osaka (3.6\% in 2006 and $5.1 \%$ in 2011; adjusted rate ratio per year, 1.03; $p$ for trend $=0.08$ ).

Conclusions: Survivals after $\mathrm{OHCA}$ were increased in Seoul while remained constant in Osaka, which may

\section{Strengths and limitations of this study}

- This study demonstrated the differences in survival outcomes between two Asian communities.

- We used data from two large population-based registries of out-of-hospital cardiac arrest (OHCA).

- Neurological and survival outcomes in Seoul increased significantly over the study period.

- Neurological and survival outcomes in Osaka changed steadily over the study period.

- The limitation is that ilnformation on hospitalbased postresuscitation care of OHCA that might affect outcomes was not available.

have been affected by the differences and improvements of patient, community, and EMS system factors.

\section{INTRODUCTION}

Out-of-hospital cardiac arrest (OHCA) is a significant global public health problem. ${ }^{1}{ }^{2}$ Despite the advances in emergency medical service (EMS) systems and resuscitation technology over the past several decades, however, only a minority of OHCA patients are successfully resuscitated and discharged with minimal neurological impairment. ${ }^{1-4}$

The temporal trends of survival outcomes after OHCA show variations across different communities. $^{2}{ }^{5-9}$ Some nationwide population-based studies have successfully demonstrated significant improvements in the chain of survival and outcomes, while others have shown no improvements over the past 20 years. ${ }^{5}{ }^{6}{ }^{10} \mathrm{~A}$ better understanding of temporal trends in survival outcomes and chain of survival may corroborate evidence-based interventions towards reducing the health burden of OHCA. 
There have been several EMS-based multicentre studies on OHCAs, ${ }^{1-14}$ reflecting different regional circumstances, cultural aspects and EMS practices of participating communities. Thereupon, numerous reports have demonstrated considerable regional variations in resuscitation outcomes of OHCA with respect to those factors. Recently, an international, multicentre, prospective registry of OHCA across the Asia-Pacific region was developed with the aim of generating best practice protocols for Asian EMS systems by reflecting on regional characteristics, and ultimately to improve OHCA survival. ${ }^{11} 14$ This ongoing international collaboration provides standardised data across different communities and enables researchers to investigate the inherent regional variations in EMS systems and OHCA outcomes. ${ }^{14}$ Understanding regional characteristics and temporal trends is critical for developing culturally appropriate interventions. ${ }^{14-16}$

The purpose of this study was to compare the temporal trends in survival outcome, chain of survival and patient factors for OHCAs between two large metropolitan communities in Asia, and to evaluate important factors that affect survival after OHCA, using populationbased registries according to the international research guidelines for OHCA.

\section{METHODS}

The Seoul-Osaka Resuscitation Study (SORS) group is a collaborating study group of the two communities' research scientists in Seoul (Korea) and Osaka (Japan). This study was carried out in those two metropolitan communities which have prospective and populationbased registry systems of OHCA. The study was approved by the institutional review boards of the Seoul National University and Osaka University. ${ }^{17}$

\section{Study setting}

The total population was 9.6 million in Seoul (2010) and 8.8 million in Osaka (2010). The population structures and EMS characteristics of the two communities are shown in table 1 .

In Korea, policies and laws for developing public education and training programmes for cardiopulmonary resuscitation (CPR) were enacted in 2002, and the actual training programme began later in 2006 with the support of the Seoul Metropolitan Government. Government-backed financial support for the supply of automatic external defibrillators (AEDs) in public places became compulsory in 2008 with the Good Samaritan Law, and was expanded to more private places in 2012. The fund was also used to support advocacy and education for high-quality bystander CPR since $2008 .^{18}$

In Osaka, CPR training for citizens has been offered since 1994, and each year approximately 120000 citizens participate in conventional CPR training. Citizens gained legal permission to use AED in July 2004, and public access defibrillators (PADs) have become increasingly available in Osaka. ${ }^{10}$

In both communities, the EMS level is intermediate where the highest qualified emergency medical technicians can give CPR with AED, perform advanced airway management and inject intravenous fluid or drug. Under each country's guideline, EMS providers are required to continue CPR unless there is a return of spontaneous circulation on the scene. In Seoul, EMS providers are encouraged to scoop and run to the emergency department (ED) while giving CPR during ambulance transport as soon as possible after giving one cycle of CPR. In Osaka, EMS providers are usually encouraged to stay for around $10 \mathrm{~min}$ for interventions including 34 cycles of CPR. ${ }^{17}$ EMS providers can withdraw provision of CPR with online medical control when there is

Table 1 Characteristics of population and EMS system in Seoul and Osaka

\begin{tabular}{|c|c|c|}
\hline & Seoul & Osaka \\
\hline Total population, $\mathrm{N}$ & 9631482 & 8776018 \\
\hline Area $\left(\mathrm{km}^{2}\right)$ & 605 & 1898 \\
\hline Population density $\left(/ \mathrm{km}^{2}\right)$ & 15914 & 4624 \\
\hline Age, year, median (IQR) & $37(23-52)$ & $43(26-63)$ \\
\hline Gender ratio (male: female) & 0.96 & 0.93 \\
\hline \multicolumn{3}{|l|}{ EMS, $N$} \\
\hline Ambulance stations & 114 & 212 \\
\hline Basic EMS providers & 382 & 1671 \\
\hline Intermittent EMS providers & 347 & 1204 \\
\hline Ambulance vehicles & 117 & 286 \\
\hline \multicolumn{3}{|l|}{ Change in community/EMS effort in improving outcomes after OHCA, year } \\
\hline Bystander CPR in EMS Act or fire department regulation & 2002 & 1982 \\
\hline $\begin{array}{l}\text { Standardisation of CPR training and public support programme for } \\
\text { CPR training for bystander and first responder }\end{array}$ & 2005 & 1993 \\
\hline PAD programme in EMS Act or fire department regulation & 2008 & 2004 \\
\hline Quality assurance for EMS performance & 2005 & 1998 \\
\hline Special continuous medical education programme for EMS providers & 2007 & 1991 \\
\hline
\end{tabular}


evident death, such as rigor mortis, postmortem lividity, incineration, decomposition or decapitation, as well as patients with 'Do Not Resuscitate' orders. All patients with OHCA who were assessed by EMS providers were transported to an ED.

An EMS system quality control programme was initiated in 1998 in Osaka through the Utstein Osaka Project, while the quality control programme in Seoul was established in 2011.9 1920 Numbers of ambulances per square kilometre were 0.19 (2010) and 0.15 (2009) in Seoul and Osaka, respectively (table 1).

\section{Study population}

Eligible patients were resuscitation-attempted OHCAs with presumed cardiac aetiology between January 2006 and December 2011. Resuscitation-attempted OHCAs were defined as those that were attempted with any resuscitation efforts, including defibrillation by a layperson or chest compression or defibrillation by EMS providers or ED healthcare workers. Patients were identified as having an arrest of cardiac aetiology by medical record review. Aetiology of arrest was presumed to be cardiac unless it was caused by cerebrovascular disease, respiratory disease, malignant tumours or external factors, or any other non-cardiac aetiology according to the international guideline for OHCA. ${ }^{21}$

\section{Data sources}

Data were collected from the EMS run sheet in Osaka, and from the EMS run sheet and hospital medical record review in Seoul. The following Utstein factors were collected: age, gender, aetiology of arrest, place of occurrence, witness, CPR and defibrillation by bystanders, prehospital initial ECG, CPR and defibrillation by EMS providers and survival outcomes. In both communities, the same definitions were used according to the Utstein data report form ${ }^{21}$ in which the details of each data set were described in previous reports. ${ }^{5} 910192022$ The elapsed time intervals, such as from call to wheel arrival at scene, from scene to departure to ED, from call to arrival at ED, and from call to first CPR, were standardised and measured in both communities. Also, time intervals from call to first defibrillation of patients with an initial shockable ECG were measured in both communities. The time intervals from call to first CPR and from call to first defibrillation were available only in 2011 in Seoul.

\section{Outcome measures}

The primary outcome was neurologically favourable survival after OHCA with cerebral performance category 1 or 2. The secondary outcome was survival to discharge (Seoul) and 1 month survival (Osaka). In Seoul, outcomes were collected by a hospital medical record review performed after discharge. The medical record review was performed by medical record experts employed at the Korea Center for Disease Controls and Prevention. In Osaka, outcomes were collected by EMS providers from hospital via a telephone interview or fax report.

\section{Statistical analyses}

Demographic characteristics of all eligible cases in the two communities were first explored. Continuous and categorical variables were compared using the Wilcoxon rank sum test and $\chi^{2}$ test, respectively. To evaluate the changes in baseline characteristics, $p$ for trend was calculated by the Cochran-Armitage test. Age-standardised and gender-standardised OHCA and survivor incidence rates per 100000 person-years for the study population were calculated to compare trends by years and communities. The sum of the 2010 Census data of Seoul and Osaka by age (decade) and gender was used as the standard population (direct standardisation method).

To assess whether survival outcomes had improved over time in the two communities, multivariable Poisson regression models were constructed. The Poisson distribution was used to directly estimate rate ratios (RR) instead of ORs to avoid its potential exaggeration. ${ }^{23} 24$ RRs for survival outcomes and 95\% CIs were calculated after adjusting for age and gender. After calculation of adjusted RR for each calendar year (from 2007 to 2011), we used the year 2006 as the reference year and multiplied the adjusted RR for each year by the observed survival rate for the reference year to obtain yearly risk-adjusted survival rates for the study period. ${ }^{24}$ These rates represent the estimated survival for each year if the patient case mix were identical to that in the reference year. ${ }^{24}$ We also evaluated calendar year as a continuous variable to obtain adjusted RRs for year-to-year survival trends. We also examined the effects of interaction between the calendar year and other potential risk factors on the main outcome by communities using the chunk test, followed by a backward elimination process for the full model, which included main exposure, potential risk factors and all interaction products. Since there was no statistically significant interaction product, we simply used the main exposure variable and potential risks for the final model.

\section{RESULTS}

During the study period, there were 18813 and 42340 EMS-assessed OHCAs in Seoul and Osaka, respectively, in which $11082(58.9 \%)$ in Seoul and 25210 (59.5\%) in Osaka were selected for analysis as resuscitationattempted OHCAs with cardiac aetiology (figure 1). The characteristics of the patients, community and EMS factors based on the Utstein criteria are shown in table 2.

The temporal trends in chain of survival (resuscitation time course) and patient factors affecting outcomes after cardiac arrest are shown in table 3. The median age of patients with OHCA was lower in Seoul (65 years) than in Osaka (75 years) in 2006, and increased to 69 (in Seoul) and 77 years (in Osaka) in 2010. The proportion of OHCAs that occurred in public places increased 
SEOUL

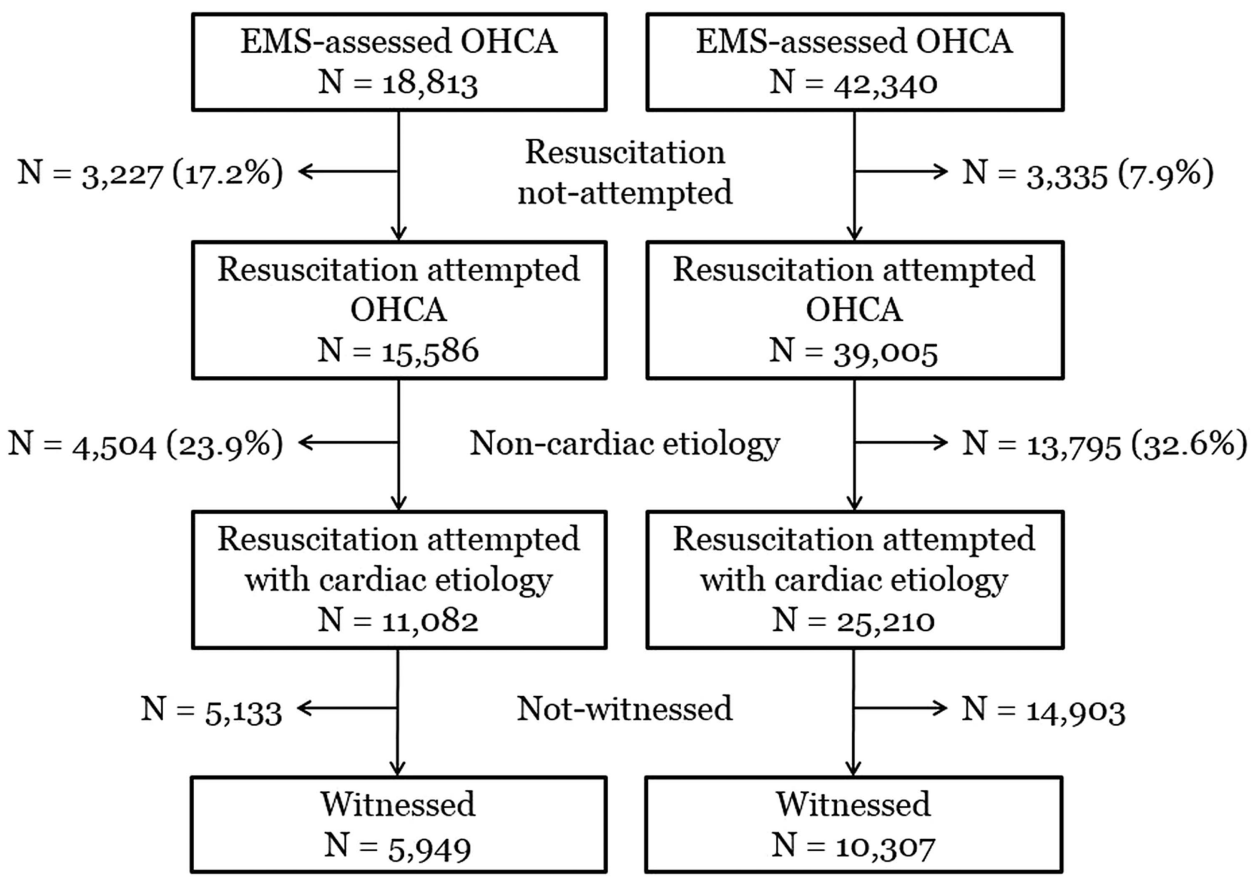

Figure 1 Study flow of out-of-hospital cardiac arrest (OHCA) patients from 1 January 2006 to 31 December 2011.

in Seoul $(12.5-20.1 \%, \mathrm{p}$ for trend $<0.01)$, whereas it decreased in Osaka $(13.5-10.5 \%$, $\mathrm{p}$ for trend $<0.01)$. In both communities, bystander CPR rates increased significantly from 2006 to 2011 (from $0.1 \%$ to $13.1 \%$ in Seoul and from $33.3 \%$ to $41.7 \%$ in Osaka). Bystander defibrillation using PAD was performed in 0.4 to $1.3 \%$ of cases annually in Osaka, while only one case was observed in Seoul due to initiation of the PAD programme later in

Table 2 Epidemiologic characteristics of resuscitation-attempted out-of hospital cardiac arrests with cardiac aetiology in Seoul and Osaka between 2006 and 2011

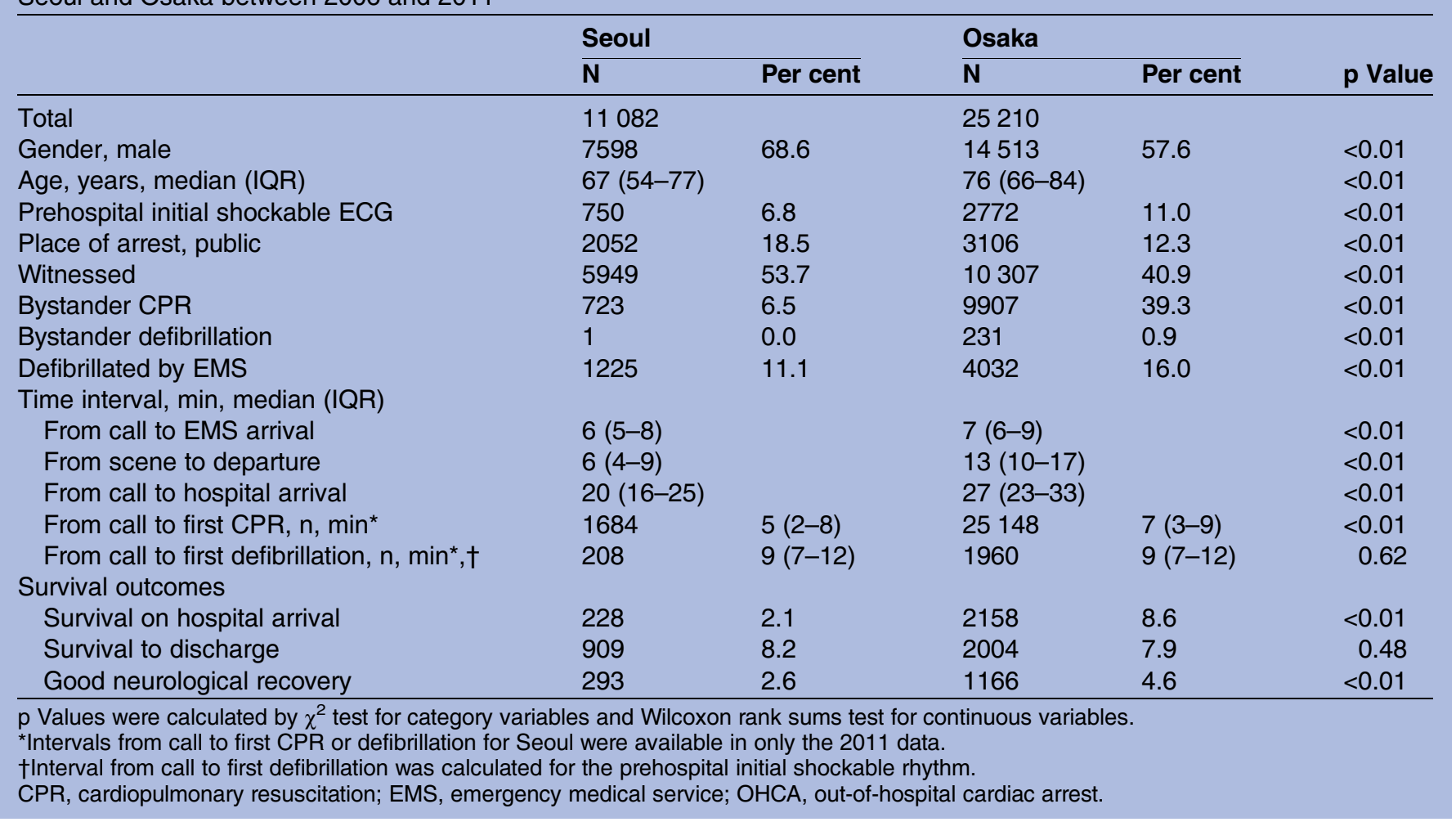




\begin{tabular}{|c|c|c|c|c|c|c|c|c|c|c|c|c|c|c|}
\hline & \multicolumn{7}{|l|}{ Seoul } & \multicolumn{7}{|c|}{ Osaka } \\
\hline & 2006 & 2007 & 2008 & 2009 & 2010 & 2011 & $p$ for trend & 2006 & 2007 & 2008 & 2009 & 2010 & 2011 & $p$ for trend \\
\hline Total, N & 1054 & 1432 & 1808 & 2107 & 2326 & 2355 & & 3559 & 3833 & 4281 & 4367 & 4477 & 4693 & \\
\hline Gender, male, \% & 66.0 & 66.6 & 69.6 & 69.9 & 68.7 & 68.7 & 0.10 & 58.7 & 55.9 & 58.8 & 57.6 & 57.6 & 56.9 & 0.40 \\
\hline Age, years, median & 65 & 66 & 66 & 66 & 68 & 69 & $<0.01$ & 75 & 76 & 76 & 77 & 77 & 77 & $<0.01$ \\
\hline Prehospital initial shockable ECG, \% & 0.1 & 2.3 & 5.3 & 7.9 & 8.3 & 11.0 & $<0.01$ & 12.6 & 11.4 & 11.7 & 11.0 & 10.3 & 9.4 & $<0.01$ \\
\hline Place of arrest, public, \% & 12.5 & 13.6 & 23.9 & 19.6 & 17.5 & 20.1 & $<0.01$ & 13.5 & 13.4 & 13.0 & 12.7 & 11.3 & 10.5 & $<0.01$ \\
\hline Witnessed, \% & 58.8 & 55.1 & 55.0 & 54.0 & 51.9 & 51.0 & $<0.01$ & 41.3 & 42.0 & 39.7 & 41.4 & 41.0 & 40.1 & 0.30 \\
\hline Bystander CPR, \% & 0.1 & 2.5 & 3.8 & 6.3 & 7.6 & 13.1 & $<0.01$ & 33.3 & 38.0 & 39.0 & 42.0 & 40.3 & 41.7 & $<0.01$ \\
\hline Bystander defibrillation, \% & 0.0 & 0.0 & 0.0 & 0.0 & 0.0 & 0.0 & 0.20 & 0.4 & 0.7 & 0.9 & 1.3 & 1.1 & 1.0 & $<0.01$ \\
\hline Defibrillated by EMS, \% & 0.4 & 6.6 & 9.1 & 11.7 & 13.1 & 17.5 & $<0.01$ & 17.7 & 17.1 & 17.4 & 16.0 & 14.8 & 13.7 & $<0.01$ \\
\hline \multicolumn{15}{|l|}{ Time interval, min, median } \\
\hline From call to EMS arrival & 6 & 6 & 6 & 6 & 7 & 6 & 0.03 & 7 & 7 & 7 & 7 & 8 & 8 & $<0.01$ \\
\hline From scene to departure & 5 & 6 & 6 & 6 & 6 & 6 & $<0.01$ & 12 & 13 & 13 & 13 & 13 & 13 & $<0.01$ \\
\hline From call to hospital arrival & 19 & 19 & 20 & 20 & 20 & 20 & $<0.01$ & 26 & 27 & 27 & 28 & 28 & 28 & $<0.01$ \\
\hline From call to first CPR & NA & NA & NA & NA & NA & 5 & & 7 & 7 & 6 & 7 & 7 & 7 & $<0.01$ \\
\hline From call to first defibrillation* & NA & NA & NA & NA & NA & 11 & & 10 & 9.5 & 10 & 9 & 10 & 10 & 0.69 \\
\hline \multicolumn{15}{|l|}{ Survival outcomes } \\
\hline Survival on hospital arrival, $\mathrm{n}$ & 13 & 21 & 31 & 47 & 46 & 70 & $<0.01$ & 259 & 359 & 367 & 361 & 370 & 442 & 0.06 \\
\hline Survival to discharge, $\mathrm{n}$ & 72 & 104 & 121 & 179 & 190 & 243 & $<0.01$ & 256 & 335 & 342 & 363 & 342 & 366 & 0.90 \\
\hline Good neurological recovery, $n$ & 15 & 35 & 45 & 52 & 52 & 94 & $<0.01$ & 127 & 204 & 202 & 213 & 196 & 224 & 0.30 \\
\hline \multicolumn{15}{|l|}{ Population measurements } \\
\hline Standardised incidence rate $†$ & 15.4 & 21.0 & 26.6 & 31.3 & 35.8 & 37.0 & & 30.6 & 32.7 & 36.5 & 36.8 & 37.4 & 39.1 & \\
\hline Standardised survivor rate $†$ & 1.0 & 1.3 & 1.4 & 2.2 & 2.5 & 3.1 & & 2.4 & 3.2 & 3.2 & 3.5 & 3.2 & 3.5 & \\
\hline
\end{tabular}


A

Resuscitation attempted OHCA with cardiac etiology

SEOUL

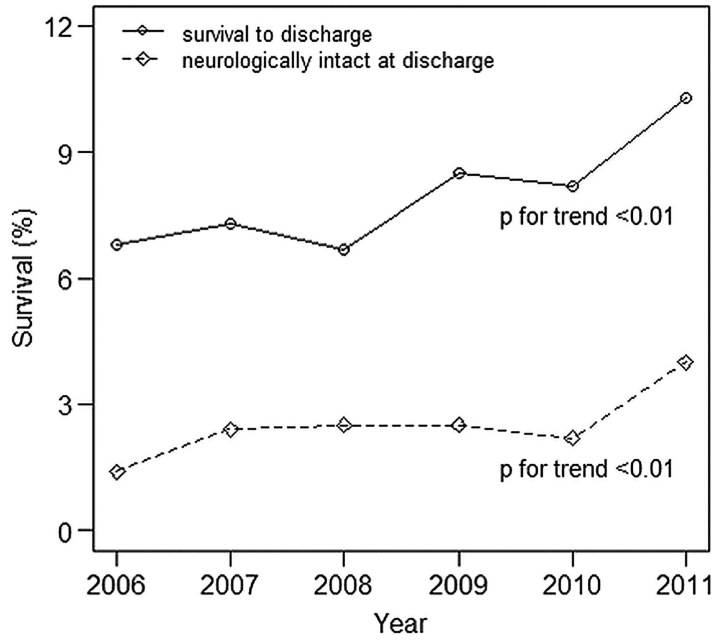

OSAKA

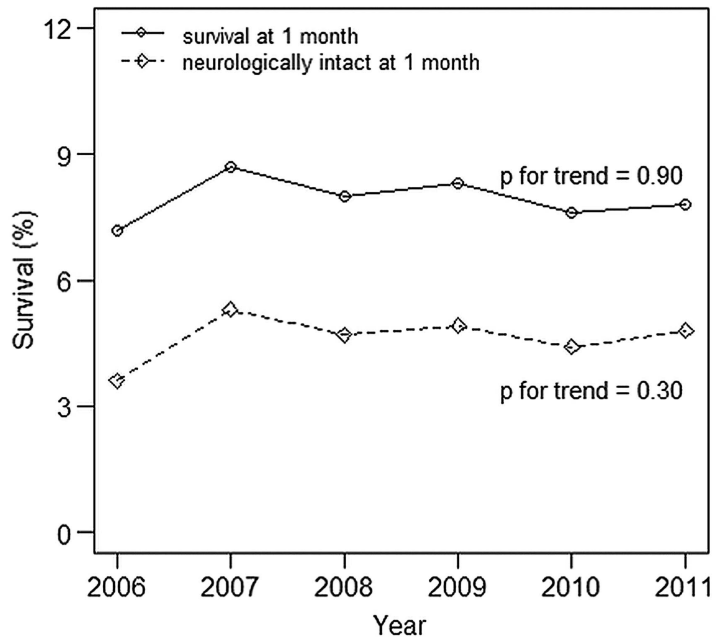

B

Witnessed and resuscitation attempted OHCA with cardiac etiology

SEOUL

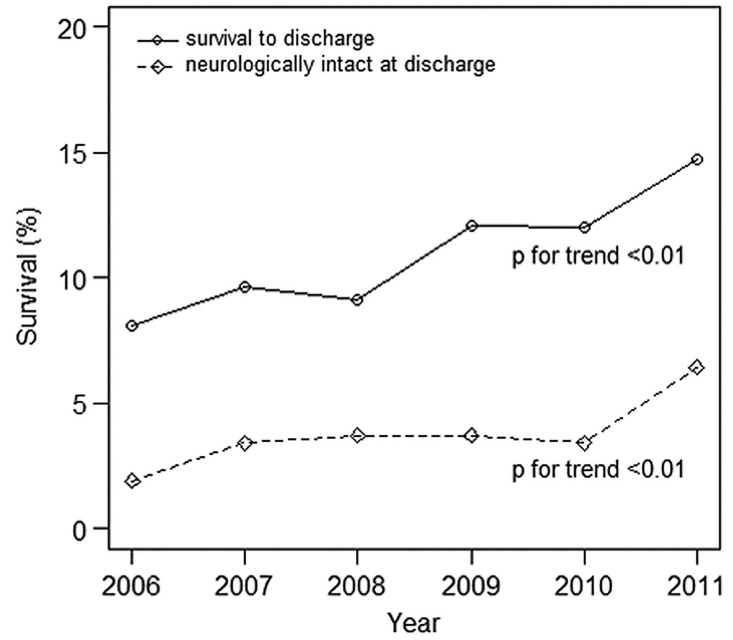

OSAKA

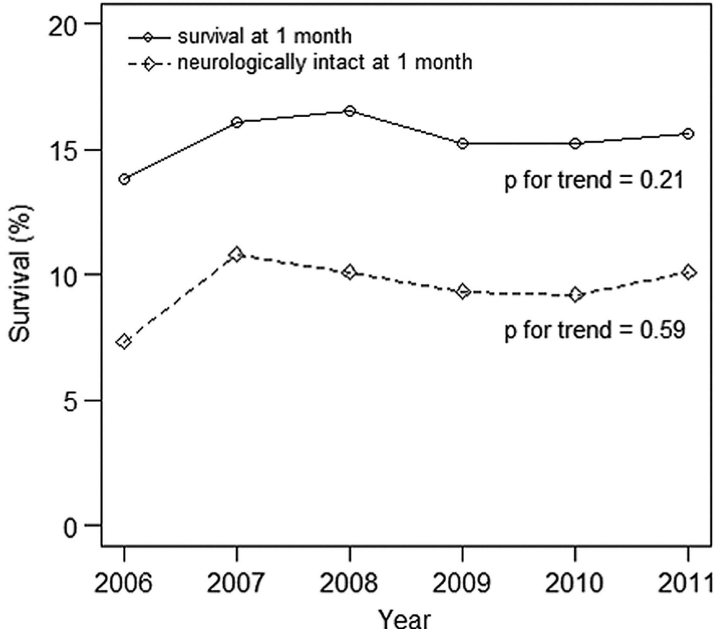

Figure 2 Temporal trends of survival outcomes by community for resuscitation attempted out-of-hospital cardiac arrest with cardiac aetiology $(A)$ and witnessed cardiac arrests $(B)$ during the study period.

2011. The proportion of OHCAs defibrillated by an EMS provider was only $0.4 \%$ in 2006 but increased to $17.5 \%$ in 2011 in Seoul, whereas the proportion in Osaka was $17.7 \%$ in 2006 followed by a slight decrease to $13.7 \%$ in 2011. Age-standardised and gender-standardised OHCA incidence rates per 100000 person-years increased in both communities during the study period from 15.4 to 37.0 in Seoul, and from 30.6 to 39.1 in Osaka. Age-and gender-standardised OHCA survival rates per 100,000 person-years also increased in both communities from 1.0 to 3.1 in Seoul and from 2.4 to 3.5 in Osaka.

Figure 2 shows temporal trends in survival and neurological outcomes in the two communities. During the study period, the overall neurologically favourable survival in resuscitation-attempted OHCAs with cardiac aetiology was $2.6 \%$ in Seoul and $4.6 \%$ in Osaka $(p<0.01)$; and the proportions in witnessed cardiac arrests were higher at $4.0 \%$ in Seoul and $9.5 \%$ in Osaka $(\mathrm{p}<0.01)$.
From 2006 to 2011, the neurologically favourable survival in OHCAs with cardiac aetiology increased significantly from $1.4 \%$ to $4.0 \%$ in Seoul ( $p$ for trend $<0.01$ ), whereas no significant temporal improvement was observed in Osaka (3.6\% in 2006 and 4.8\% in 2011; p for trend $=0.30$ ). Rates of survival to discharge increased significantly in Seoul from $6.8 \%$ in 2006 to $10.3 \%$ in 2011 ( $\mathrm{p}$ for trend $<0.01$ ), while no significant increase was observed in Osaka (7.2\% in 2006 and $7.8 \%$ in 2011; $\mathrm{p}$ for trend=0.90) (figure 2A). In the subpopulation of witnessed resuscitation-attempted OHCAs, similar regional trends were observed as in all OHCAs with cardiac aetiology; in Seoul, both survival and neurological outcomes were significantly enhanced, while no significant increase was observed in Osaka (figure 2B).

Table 4 shows risk-adjusted temporal trends in survival outcomes in the two communities. Age-adjusted and gender-adjusted rates of neurologically favourable 
Table 4 Risk-adjusted temporal trends in survival outcomes by community

\begin{tabular}{|c|c|c|c|c|c|c|c|c|c|c|}
\hline & \multicolumn{6}{|c|}{ Risk-adjusted survival rate*, \% } & \multicolumn{3}{|c|}{$\begin{array}{l}\text { Adjusted rate ratio per } \\
\text { yeart }\end{array}$} & \multirow[b]{2}{*}{$\mathrm{p}$ For trend $\boldsymbol{t}$} \\
\hline & 2006 & 2007 & 2008 & 2009 & 2010 & 2011 & $\overline{\mathbf{R R}}$ & $95 \% \mathrm{C}$ & & \\
\hline \multicolumn{11}{|l|}{ Total } \\
\hline \multicolumn{11}{|c|}{ Good neurological outcome } \\
\hline Seoul & 1.4 & 2.5 & 2.5 & 2.5 & 2.4 & 4.3 & 1.17 & 1.09 & 1.26 & $<0.01$ \\
\hline Osaka & 3.6 & 5.5 & 4.8 & 5.1 & 4.7 & 5.1 & 1.03 & 1.00 & 1.07 & 0.08 \\
\hline \multicolumn{11}{|c|}{ Survival to discharge } \\
\hline Seoul & 6.8 & 7.3 & 6.7 & 8.5 & 8.6 & 10.9 & 1.10 & 1.06 & 1.15 & $<0.01$ \\
\hline Osaka & 7.2 & 9.0 & 8.1 & 8.6 & 8.0 & 8.2 & 1.01 & 0.98 & 1.03 & 0.53 \\
\hline \multirow{2}{*}{\multicolumn{11}{|c|}{$\begin{array}{l}\text { Witnessed } \\
\text { Good neurological outcome }\end{array}$}} \\
\hline & & & & & & & & & & \\
\hline Seoul & 1.9 & 3.5 & 3.7 & 3.8 & 3.7 & 6.9 & 1.21 & 1.11 & 1.32 & $<0.01$ \\
\hline Osaka & 7.3 & 10.6 & 10.0 & 9.4 & 9.4 & 10.2 & 1.03 & 0.99 & 1.07 & 0.13 \\
\hline \multicolumn{11}{|c|}{ Survival to discharge } \\
\hline Seoul & 8.1 & 9.8 & 9.1 & 12.4 & 12.8 & 15.5 & 1.14 & 1.08 & 1.19 & $<0.01$ \\
\hline Osaka & 13.8 & 16.0 & 16.3 & 15.3 & 15.5 & 15.8 & 1.01 & 0.98 & 1.04 & 0.43 \\
\hline
\end{tabular}

survival in resuscitation-attempted OHCAs with cardiac aetiology increased significantly in Seoul from $1.4 \%$ in 2006 to $4.3 \%$ in 2011 (adjusted RR per year, 1.17; 95\% CI 1.09 to 1.26 ; $\mathrm{p}$ for trend $<0.01$ ), while no significant improvements were observed in Osaka (from $3.6 \%$ in 2006 to $5.1 \%$ in 2011; adjusted RR per year, 1.03 ; $95 \%$ CI 1.00 to $1.07 ; \mathrm{p}$ for trend $=0.08)$. In witnessed cardiac arrests, age-adjusted and gender-adjusted rates of neurologically favourable survival increased significantly from $1.9 \%$ to $6.9 \%$ in Seoul (adjusted RR per year, $1.21 ; 95 \%$ CI 1.11 to 1.32 ; $\mathrm{p}$ for trend $<0.01$ ), whereas no significant change was observed in Osaka (adjusted RR per year, 1.03 ; $95 \%$ CI 0.99 to 1.07 ; $\mathrm{p}$ for trend $=0.13$ ).

\section{DISCUSSION}

This study demonstrated the differences in temporal trends in survival outcomes between two Asian communities and their associated factors for improving survival after OHCA using data from two large population-based registries of OHCA. Neurologically favourable survival in Seoul was significantly increased over the study period, but still marked relatively low rates. On the other hand, while showing an insignificant and steady change over the years, the overall survival rates remained relatively high in Osaka. We found difficulty in improving survival after OHCA in communities with already existing and developed EMS systems in places, such as Osaka, compared with communities with a developing EMS system, such as Seoul.

Between 2006 and 2011, there have been substantial improvements at the community level and in the EMS system to increase survival outcomes of OHCA in Seoul. Bystander CPR education and advertising campaign were diffused quickly, ${ }^{18}$ and the proportion of bystander CPR increased rapidly (about 13\%) within the 6-year period of this study. Bystander CPR can double the chance of survival from an OHCA event in previous studies. ${ }^{25}{ }^{26}$ For EMS providers, education programmes and quality control protocols were developed. In line with these efforts, initial ECG check and application of AEDs by EMS providers improved from 5\% in 2006 to $45 \%$ in 2011 (data not shown), thereby leading to an increase in the proportion of initial shockable ECG from $0.1 \%$ in 2006 to $11.0 \%$ in 2011 and defibrillation by EMS from $0.4 \%$ in 2006 to $17.7 \%$ in 2011. Initial rhythms of ventricular fibrillation have previously been associated with enhanced survival outcomes. ${ }^{2}{ }^{27}$ In Seoul, median time intervals from call to initial CPR and initial defibrillation were 5 and $11 \mathrm{~min}$, respectively, in 2011, which were similar to those observed in Osaka. Although the resuscitation time course before 2011 was not applicable in Seoul, we assume that it would have been shortened during the study period. In accordance with these improvements in the chain of survival, the survival outcomes of OHCAs in Seoul were significantly improved throughout the study period (table 4).

However, in Osaka, neurologically favourable survival did not increase during the study period. Numerous efforts to improve EMS factors, such as bystander CPR and prehospital defibrillation by EMS personnel, had already been implemented in the early 2000s in Osaka. Accordingly, a previous study reported significant improvements in bystander CPR, decreased time intervals from collapse to first CPR and first defibrillation, and improvements in survival outcomes during 1998 and 2006 in Osaka. ${ }^{5}$ In addition, OHCA incidence in public places and bystander-witnessed rates were 
decreased, which may have been characterised by the ageing society of Osaka. Despite continuous efforts in improving EMS factors and maintaining a relatively good EMS system in place, the observed plateau in the survival rates of Osaka may have accounted for a limit of obtainable benefits from the current EMS basic-to-intermediate level.

Nevertheless, survival in the two metropolitan communities is still less than optimal, suggesting the need to address persistent issues in EMS factors. The PAD programme in Osaka has been more readily available since 2004; however, the proportion of bystander defibrillation still remains low. Only $0.9 \%$ received bystander defibrillation despite the $11 \%$ of OHCAs having initial shockable rhythm. While more than 300000 PADs were distributed for use in Japan, the proportion of prehospital defibrillation by laypersons was still less than $3 \%$ of OHCAs. ${ }^{10}$ Further adaptation of the PAD programme, such as distribution of neighbourhood-accessible AEDs and widespread deployment of home-based AEDs, may save more lives, although its effectiveness is still controversial. Redistribution of PADs based on the coverage rate of OHCA and a coordinated PAD programme including AED networks should also be considered. ${ }^{28} 29$ Furthermore, proportions of bystander CPR in the two Asian communities were significantly lower compared with other countries such as Norway $(76 \%$ for resuscitation-attempted OHCA with cardiac aetiology) or Sweden (59\% for witnessed OHCA). ${ }^{30} 31$ Dispatcherassisted CPR, practice-based CPR training and a public awareness campaign to promote CPR and AEDs have been shown to improve CPR and AED use by bystanders. 1011182631

As the quality of CPR is crucial to improve survival after OHCA, an effective and efficient CPR education programme and EMS quality control protocol, including a real-time feedback programme, should be considered. ${ }^{32}{ }^{33}$ Modification of the EMS protocol to improve the quality of CPR on ambulance during transport should also be given consideration.

\section{Limitations}

This study has some limitations. Since this study was performed in two different metropolitan communities with basic-to-intermediate EMS service levels, we cannot generalise the results to communities with different EMS systems. Second, the outcomes in the two communities were measured at different times (at discharge in Seoul, and at 1 month in Osaka). Furthermore, information on hospital-based postresuscitation care of OHCA was not available, which may serve as an important factor in survival outcomes. Finally, while we tried to classify the patients using standardised definitions based on international guidelines, ${ }^{21}$ possible misclassification may have occurred, including the definition of cardiac aetiology, which can vary depending on the rigour of the efforts to identify other causes.

\section{CONCLUSION}

In two large metropolitan communities in Asia, the temporal trends in survival outcome and associated factors for improving survival after OHCA were different. In response to an enhancement in the chain of survival, the survival outcomes after OHCA were significantly increased in Seoul, while these remained steady in Osaka despite ongoing regional efforts to improve community and EMS factors.

Author affiliations

${ }^{1}$ Laboratory of Emergency Medical Services, Seoul National University Hospital Biomedical Research Institute, Seoul, Korea

${ }^{2}$ Department of Emergency Medicine, Seoul National University College of Medicine, Seoul, Korea

${ }^{3}$ Division of Environmental Medicine and Population Sciences, Department of Social and Environmental Medicine, Osaka University Graduate School of

Medicine, Suita, Japan

${ }^{4}$ Department of Traumatology and Acute Critical Medicine, Osaka University Graduate School of Medicine, Suita, Japan

${ }^{5}$ Department of Pharmacoepidemiology, Kyoto University Graduate School of Medicine and Public Health, Kyoto, Japan

${ }^{6}$ Department of Acute Medicine, Faculty of Medicine, Kinki University, OsakaSayama, Japan

${ }^{7}$ Senri Critical Care Medical Center, Osaka Saiseikai Senri Hospital, Suita, Japan

${ }^{8}$ Department of Health Service, Kyoto University, Kyoto, Japan

Contributors YSR analysed the data and wrote the paper; SDS and TI designed, analysed and interpreted the data and revised the paper; TK, KJS, EJL and KK analysed and interpreted the data and revised the paper; and SYK, CN, TS, TN and YH analysed the data and revised the paper.

Funding This study was supported by the Japan Pfizer Health Research Foundation and the Korea Centers for Disease Control and Prevention.

\section{Competing interests None declared.}

Ethics approval The study was approved by the institutional review boards of the Seoul National University and Osaka University.

Provenance and peer review Not commissioned; externally peer reviewed.

Data sharing statement YSR, SDS, TK, and TI had full access to all of the data in the study and takes responsibility for the integrity of the data and the accuracy of the data analysis.

Open Access This is an Open Access article distributed in accordance with the Creative Commons Attribution Non Commercial (CC BY-NC 4.0) license, which permits others to distribute, remix, adapt, build upon this work noncommercially, and license their derivative works on different terms, provided the original work is properly cited and the use is non-commercial. See: http:// creativecommons.org/licenses/by-nc/4.0/

\section{REFERENCES}

1. Mozaffarian D, Benjamin EJ, Go AS, et al. Heart disease and stroke statistics-2015 update: a report from the American Heart Association. Circulation 2015;131:e29-322.

2. Berdowski J, Berg RA, Tijssen JG, et al. Global incidences of out-of-hospital cardiac arrest and survival rates: systematic review of 67 prospective studies. Resuscitation 2010;81:1479-87.

3. Wissenberg M, Lippert FK, Folke F, et al. Association of national initiatives to improve cardiac arrest management with rates of bystander intervention and patient survival after out-of-hospital cardiac arrest. JAMA 2013;310:1377-84.

4. Chan PS, McNally B, Tang F, et al. Recent trends in survival from out-of-hospital cardiac arrest in the United States. Circulation 2014;130:1876-82.

5. Iwami T, Nichol G, Hiraide A, et al. Continuous improvements in "chain of survival" increased survival after out-of-hospital cardiac arrests: a large-scale population-based study. Circulation 2009;119:728-34. 
6. Kuisma M, Repo J, Alaspaa A. The incidence of out-of-hospital ventricular fibrillation in Helsinki, Finland, from 1994 to 1999. Lancet 2001;358:473-4.

7. Adielsson A, Hollenberg J, Karlsson T, et al. Increase in survival and bystander CPR in out-of-hospital shockable arrhythmia: bystander CPR and female gender are predictors of improved outcome. Experiences from Sweden in an 18-year perspective. Heart 2011;97:1391-6.

8. Kitamura T, Iwami T, Kawamura T, et al. Nationwide improvements in survival from out-of-hospital cardiac arrest in Japan. Circulation 2012;126:2834-43.

9. Ro YS, Shin SD, Song KJ, et al. A trend in epidemiology and outcomes of out-of-hospital cardiac arrest by urbanization level: a nationwide observational study from 2006 to 2010 in South Korea. Resuscitation 2013;84:547-57.

10. Kitamura T, Iwami T, Kawamura T, et al. Nationwide public-access defibrillation in Japan. N Engl J Med 2010;362:994-1004.

11. Ong ME, Shin SD, Tanaka $\mathrm{H}$, et al. Pan-Asian Resuscitation Outcomes Study (PAROS): rationale, methodology, and implementation. Acad Emerg Med 2011;18:890-7.

12. McNally B, Robb R, Mehta M, et al. Out-of-hospital cardiac arrest surveillance-Cardiac Arrest Registry to Enhance Survival (CARES), United States, October 1, 2005-December 31, 2010. MMWR Surveill Summ 2011;60:1-19.

13. Morrison LJ, Nichol G, Rea TD, et al. Rationale, development and implementation of the Resuscitation Outcomes Consortium Epistry-Cardiac Arrest. Resuscitation 2008;78:161-9.

14. Shin SD, Ong ME, Tanaka $\mathrm{H}$, et al. Comparison of emergency medical services systems across Pan-Asian countries: a Web-based survey. Prehosp Emerg Care 2012;16:477-96.

15. Nichol G, Thomas E, Callaway $\mathrm{CW}$, et al. Regional variation in out-of-hospital cardiac arrest incidence and outcome. JAMA 2008;300:1423-31

16. McNally B, Stokes A, Crouch A et al CARES: Cardiac arrest registry to enhance survival. Ann Emerg Med 2009;54:674-83 e2.

17. Shin SD, Kitamura T, Hwang SS, et al. Association between resuscitation time interval at the scene and neurological outcome after out-of-hospital cardiac arrest in two Asian cities. Resuscitation 2014;85:203-10.

18. Lee MJ, Hwang SO, Cha KC, et al. Influence of nationwide policy on citizens' awareness and willingness to perform bystander cardiopulmonary resuscitation. Resuscitation 2013;84:889-94.

19. Shin SD, Ahn KO, Song KJ, et al. Out-of-hospital airway management and cardiac arrest outcomes: a propensity score matched analysis. Resuscitation 2012;83:313-19.

20. Kajino K, Iwami T, Kitamura T, et al. Comparison of supraglottic airway versus endotracheal intubation for the pre-hospital treatment of out-of-hospital cardiac arrest. Critical care 2011;15:R236.

21. Jacobs I, Nadkarni V, Bahr J, et al. Cardiac arrest and cardiopulmonary resuscitation outcome reports: update and simplification of the Utstein templates for resuscitation registries: a statement for healthcare professionals from a task force of the International Liaison Committee on Resuscitation (American Heart Association, European Resuscitation Council, Australian Resuscitation Council, New Zealand Resuscitation Council, Heart and Stroke Foundation of Canada, InterAmerican Heart Foundation, Resuscitation Councils of Southern Africa). Circulation 2004;110:3385-97.

22. Shin SD, Suh GJ, Ahn KO, et al. Cardiopulmonary resuscitation outcome of out-of-hospital cardiac arrest in low-volume versus high-volume emergency departments: an observational study and propensity score matching analysis. Resuscitation 2011;82:32-9.

23. Zou G. A modified Poisson regression approach to prospective studies with binary data. Am J Epidemiol 2004;159:702-6.

24. Girotra S, Nallamothu BK, Spertus JA, et al. Trends in survival after in-hospital cardiac arrest. N Engl J Med 2012;367:1912-20.

25. Sasson C, Meischke H, Abella BS, et al. Increasing cardiopulmonary resuscitation provision in communities with low bystander cardiopulmonary resuscitation rates: a science advisory from the American Heart Association for healthcare providers, policymakers, public health departments, and community leaders. Circulation 2013;127:1342-50.

26. Sasson C, Rogers MA, Dahl J, et al. Predictors of survival from out-of-hospital cardiac arrest: a systematic review and meta-analysis. Circ Cardiovasc Qual Outcomes 2010;3:63-81.

27. Soholm H, Hassager C, Lippert F, et al. Factors associated with successful resuscitation after out-of-hospital cardiac arrest and temporal trends in survival and comorbidity. Ann Emerg Med 2014;65:523-531.e2.

28. Hansen CM, Lippert FK, Wissenberg M, et al. Temporal trends in coverage of historical cardiac arrests using a volunteer-based network of automated external defibrillators accessible to laypersons and emergency dispatch centers. Circulation 2014; 130:1859-67.

29. Folke F, Lippert FK, Nielsen SL, et al. Location of cardiac arrest in a city center: strategic placement of automated external defibrillators in public locations. Circulation 2009;120:510-17.

30. Heradstveit BE, Sunde K, Sunde GA, et al. Factors complicating interpretation of capnography during advanced life support in cardiac arrest-a clinical retrospective study in 575 patients. Resuscitation 2012;83:813-18.

31. Stromsoe A, Andersson B, Ekstrom L, et al. Education in cardiopulmonary resuscitation in Sweden and its clinical consequences. Resuscitation 2010;81:211-16.

32. Lund-Kordahl I, Olasveengen TM, Lorem T, et al. Improving outcome after out-of-hospital cardiac arrest by strengthening weak links of the local Chain of Survival; quality of advanced life support and post-resuscitation care. Resuscitation 2010;81:422-6.

33. Ma MH, Chiang WC, Ko PC, et al. Outcomes from out-of-hospital cardiac arrest in Metropolitan Taipei: does an advanced life support service make a difference? Resuscitation 2007; 74:461-9. 